\title{
How greater mouse-eared bats deal with ambiguous echoic scenes
}

\author{
M. L. Melcón · Y. Yovel • A. Denzinger • \\ H.-U. Schnitzler
}

Received: 26 February 2010 / Revised: 30 June 2010 / Accepted: 10 July 2010 / Published online: 23 July 2010

(C) The Author(s) 2010. This article is published with open access at Springerlink.com

\begin{abstract}
Echolocating bats have to assign the received echoes to the correct call that generated them. Failing to do so will result in the perception of virtual targets that are positioned where there is no actual target. The assignment of echoes to the emitted calls can be ambiguous especially if the pulse intervals between calls are short and kept constant. Here, we present first evidence that greater mouse-eared bats deal with ambiguity by changing the pulse interval more often, in particular by reducing the number of calls in the terminal group before landing. This strategy separates virtual targets from real ones according to their change in position. Real targets will always remain in a constant position, and virtual targets will jitter back and forth according to the change in the time interval.
\end{abstract}

Keywords Greater mouse-eared bats · Echolocation . Range ambiguity $\cdot$ Approach $\cdot$ Temporal pattern
Abbreviations
PI Pulse interval
SL Source level

M.L. Melcón and Y. Yovel contributed equally to this work.

M. L. Melcón $(\varangle)$ · Y. Yovel · A. Denzinger · H.-U. Schnitzler

Tierphysiologie, Institut für Neurobiologie,

Universität Tübingen, Auf der Morgenstelle 28,

72076 Tübingen, Germany

e-mail: marumelcon@gmail.com

M. L. Melcón

Marine Physical Laboratory,

Scripps Institution of Oceanography,

University of California, San Diego,

9500 Gilman Drive, La Jolla, CA 92093, USA
SPL Sound pressure level

TWTT Two-way travel time

\section{Introduction}

Echolocating bats adapt the structure and temporal pattern of the emitted calls according to the echoic scene they undergo, both in spatial orientation-related tasks as well as during foraging (reviewed in Schnitzler et al. 2003). A well-known example comprises the approach to a target, where bats change the signal structure, decrease its duration, and increase the information flow by reducing the pulse interval (PI) between calls throughout the approach. Typically calls are emitted synchronized with wing-beat and breathing cycle (Kalko and Schnitzler 1998; Wong and Waters 2001). The behavioural situation a bat is engaged in can be classified according to its echolocation characteristics: During search phase bats often emit single calls or pairs within a wing-beat cycle. During the approach to a target, calls are often grouped into clusters. The closer the bat gets to the target the higher is the number of calls in the groups and the shorter are the pulse intervals between calls. Prior to the contact with the target, bats end the sequence emitting a last group (terminal part of the approach phase or buzz) that often contains the highest number of calls (Griffin et al. 1960; Schnitzler et al. 1987; Schnitzler and Kalko 2001; Schnitzler et al. 2003; Melcón et al. 2007).

Lately several studies addressed different issues of the temporal pattern in echolocation sequences. These studies showed that echolocation behaviour does not only depend on the distance to the target alone, but also on the nature of the target of interest (e.g. flying insects, landing site: Melcón et al. 2007) and the echoic scenes bats are confronted with (e.g. presence or absence of nearby 
background: e.g. Moss and Surlykke 2001; Siemers and Schnitzler 2004; Moss et al. 2006, Petrites et al. 2009). For instance, Moss et al. (2006) found that the temporal emission pattern of Eptesicus fuscus approaching flying insects differed according to the distance between prey and background. The last group of the sequence was shorter when the prey was close to a reflective background compared to terminal groups emitted when pursuing prey in an open room. This means that bats not only adapt their echolocation behaviour to the distance of the target of interest, but there is also a reaction to additional objects behind the target in focus.

In such situations, where more than one object is present, bats need to evaluate the relation of an emitted call to an echo train containing echoes from more than just a single object. In these cases, the correct assignment of the received echoes to the corresponding emitted calls may be crucial (Skolnik 1970). This might sound like a trivial task, but when emitting calls with short and nearly constant PIs between them, as it is often the case when approaching a target, echolocating animals are prone to face two sources of confusion: ambiguity type I occurs when the echo of the last call is assigned to the former call. This leads to an overestimation of the range. Ambiguity type II occurs when the echo of a previous call is assigned to the last call. This leads to an underestimation of the range (Fig. 1). In both cases the wrong assignment leads to the perception of a "virtual object" which stays at the same position as long as the PI is constant. In RADAR systems virtual echoes are identified by an alternation of the pulse interval from short to long and vice versa thus avoiding constant pulse intervals. This alternation induces a range jitter of the virtual target but not of the real target.

Theoretically there are three possibilities to avoid ambiguity type II and reduce the probability of ambiguity type I: (1) an increase of the pulse interval in the presence of further reflectors would make sure that all echoes are back before the next call is emitted (Holderied and

a
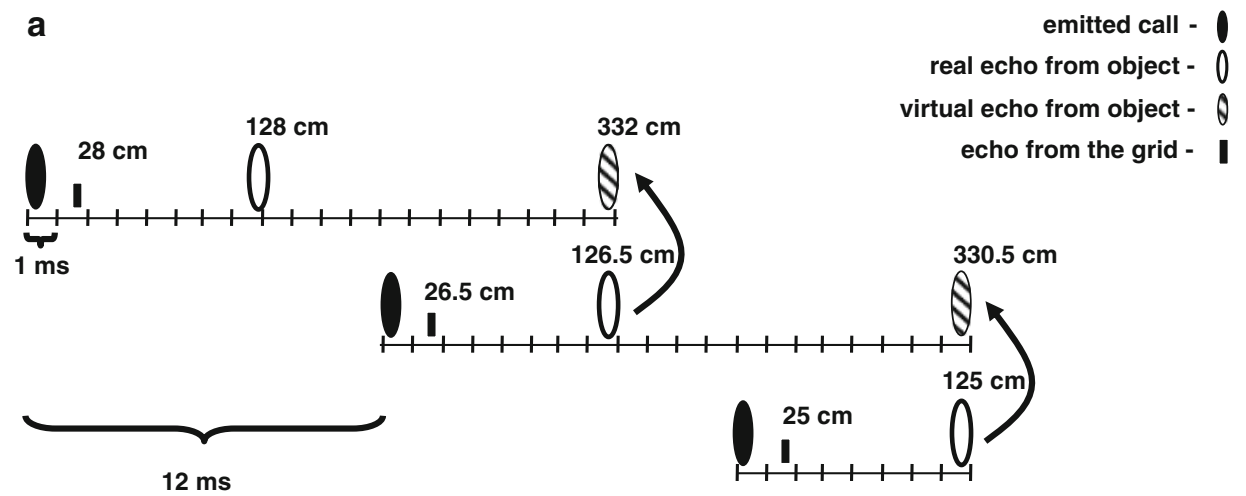

b

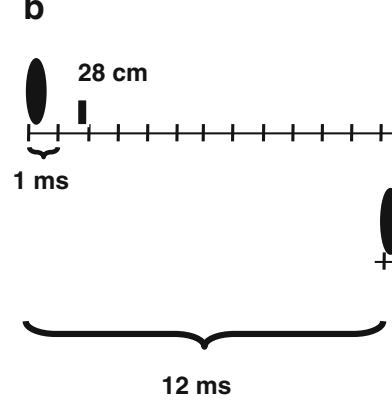

$278 \mathrm{~cm}$ 
von Helversen 2003); (2) alternation of long and short PIs would prevent both types of ambiguity by being able to identify virtual targets (Skolnik 1970); and/or (3) marking consecutive calls by alternating any of their echolocation parameters in a way that eases the echo assignment (Kingston et al. 2003; Hiryu et al. 2010).

In bats, the probability for ambiguity type I is small at large pulse intervals. In this situation the virtual target would be perceived as being far away at a distance far beyond the detection range of the bat. In addition, even if the virtual object were within the detection range, spectral cues (low-pass filtering of the echo) should help to reveal its ambiguousness. These filtering effects weaken the shorter the PI is. The probability for ambiguity type I therefore increases when the PI becomes shorter during the approach to an object and is the highest in the terminal group. Ambiguity type II occurs if the two-way travel time of a target echo is larger than the pulse interval. In echoic scenes where the target of interest and the background target are close together ambiguity type II only occurs at the short pulse intervals within the groups of the approach phase. In this situation the virtual target distance is shorter than the real one so that bats should have no problem to recognize that the virtual target echo has a lower amplitude and is stronger lowpass filtered as the virtual distance predicts and thus cannot be real.

Although ambiguity is a general problem potentially relevant to all echolocating animals, it has been poorly studied and its relevance along with the ways to deal with it remains unclear. Ambiguity type I has never been discussed in any echolocating animal yet. Ambiguity type II has been discussed in the context of maximum detection ranges of bats flying in open space but bats looking for targets far away usually do not emit a call before receiving all echoes from the previous one (Schnitzler and Kalko 2001; Holderied and von Helversen 2003; Holderied et al. 2005). However, only lately a study has been carried out with Eptesicus fuscus where the target of interest and background are close together and where the pulse intervals are short enough to cause ambiguity type II (Hiryu et al. 2010). In this situation the bats marked consecutive calls by alternating their terminal frequency so that the echo assignment was possible.

In this paper we tested how greater mouse-eared bats (Myotis myotis) deal with potentially ambiguous echoic scenes when landing. By positioning an additional background object at 100 or $250 \mathrm{~cm}$ behind the landing grid we produced echo trains which were prone to cause both types of ambiguity in echolocating bats. Besides we ensonified the system to verify whether bats could have used the spectral cues available in the echoes to identify them and thus avoid the ambiguity problem.

\section{Materials and methods}

Animals

We used four adult male greater mouse-eared bats (M. myotis) from Bulgaria to perform the experiments. We kept them in a room with constant temperature $\left(24 \pm 2{ }^{\circ} \mathrm{C}\right.$ temperature $)$ and humidity $(65 \pm 5 \%)$. The light:dark cycle was of 16:8 h. The bats had free access to fresh water and ate mealworms (larvae of Tenebrio molitor) only during the experimental sessions. We supplemented the diet with vitamins (Nutri$\mathrm{cal}^{\circledR}$, Albrecht) and minerals (Korvimin ${ }^{\circledR}$, WDT).

\section{Experimental setup}

We conducted all experiments in a flight room $(3.6 \times 6.0 \times 2.8 \mathrm{~m})$ covered with acoustic foam in order to reduce reverberations. Bats were trained to take off from the hand at a distance of $100 \mathrm{~cm}$ from a vertical grid $(10 \times 12 \mathrm{~cm})$ located in the center of the room, and land on it. An ultrasonic microphone was placed right behind (ca. $5 \mathrm{~cm}$ ) the landing platform to pick up the echolocation calls. We assumed that during training bats adjusted their behaviour such that they can correctly assign the grid echoes. The echolocation behaviour of these flights was used as a control and referred to as the control behaviour.

To enhance ambiguity type I we positioned a vertical cardboard $(70 \times 130 \mathrm{~cm})$ covered with aluminium $100 \mathrm{~cm}$ behind the grid. Its echoes returned $\sim 6 \mathrm{~ms}$ after that of the landing grid. This reflector was too close to create ambiguity type II, since the time delay between signal and echo was always shorter than the pulse interval. It was close enough though, that with a short PI its echo could be assigned to the previous pulse. For instance, if the bat was $20 \mathrm{~cm}$ from the grid and the PIs were $\sim 14 \mathrm{~ms}$ a false assignment would result in a virtual object at $\sim 360 \mathrm{~cm}$ relative to the bat. To increase ambiguity type II we positioned the same reflector $250 \mathrm{~cm}$ behind the grid. When the bat was close to the landing grid, the time delay of its echo was often larger than the PI (Fig. 2), thus generating ambiguity type II. For instance, a bat emitting signals with a PI of $14 \mathrm{~ms} 20 \mathrm{~cm}$ from the grid would hear the echo from the reflector after the emission of the next pulse. If the echo was assigned to the latest emitted pulse, a virtual object $32 \mathrm{~cm}$ from the bat would be generated. This reflector could also induce ambiguity type I resulting in a virtual target at about $5.10 \mathrm{~m}$; however, this virtual object would probably be too far out to be of importance.

\section{Data recording and analysis}

We recorded the echolocation signals using custom-made equipment (PCTape, Department of Animal Physiology, 
Fig. 2 a PIs along the distance to the landing grid of all trials for each bat. Treatments are depicted as follows: black circles control, red circles object at $100 \mathrm{~cm}$, green circles object at $250 \mathrm{~cm}$. Red lines represent the two-way travel time of the sound impinging the reflector at $100 \mathrm{~cm}$ and green lines at $250 \mathrm{~cm}$. b Means \pm SEM of the PI within groups for each distance class. For this, the whole approach has been divided into 8 equal distance classes. Only bat 1 increased the PI slightly along the entire approach when a reflector was present behind the landing grid (colour figure online)
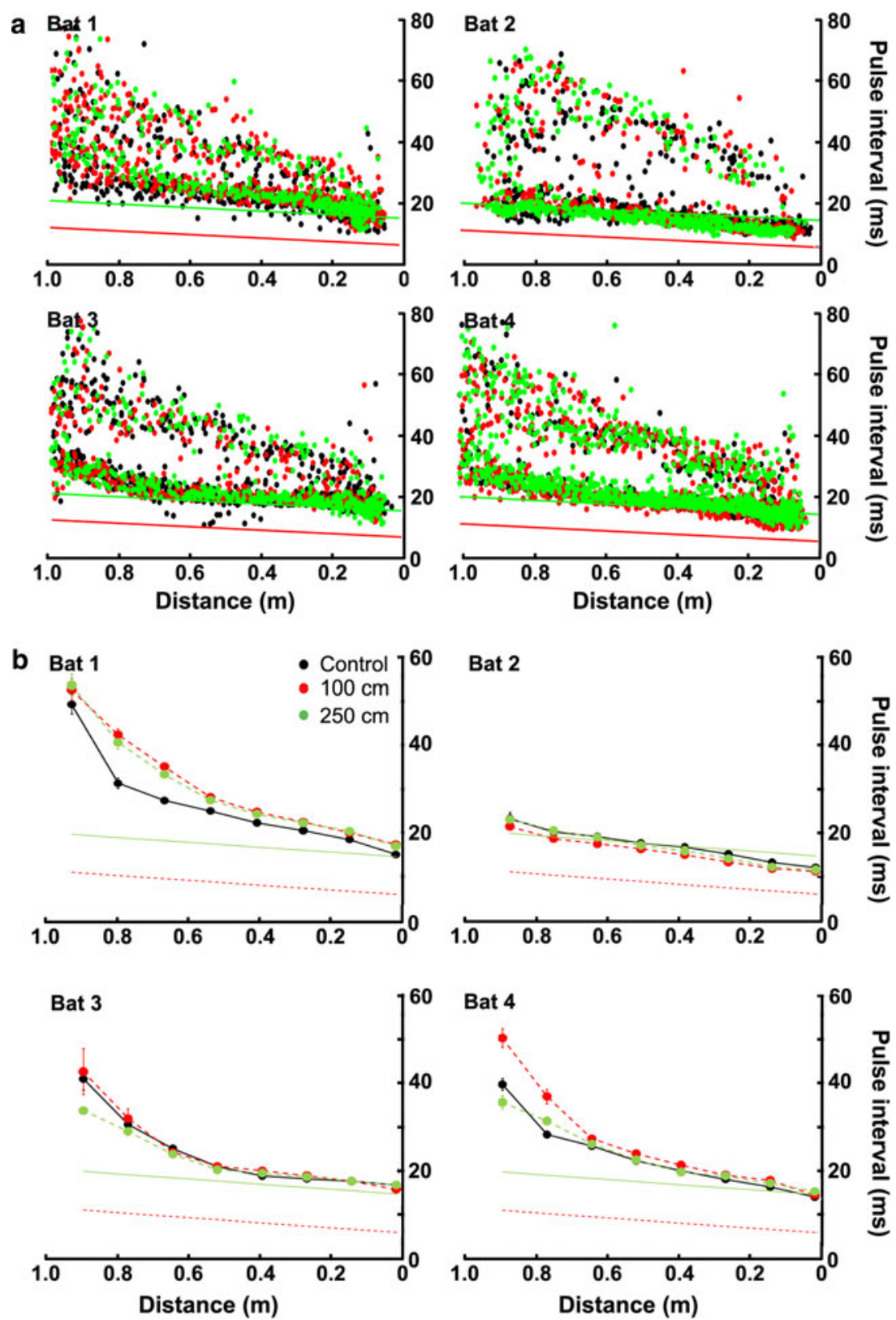

University of Tübingen). The echolocation pulses were picked up with a custom-made ultrasonic microphone [flat frequency response $( \pm 3 \mathrm{~dB}$ ) between 18 and $200 \mathrm{kHz}$, digitized with a sampling rate of $480 \mathrm{kHz}$ and a resolution of 16 bits, and stored as wav-files. The bats' trajectories were recorded with two IR-cameras (Sanyo IRP; 50 half frames $\mathrm{s}^{-1}$ ) fixed in two upper corners of the room. Each half frame was illuminated for $1 \mathrm{~ms}$ by two infrared stroboscopic units placed on the floor. The video recordings were stored on Panasonic DVC Mini video tapes using two Sony camcorders (TRV 30E). Video and sound recordings were synchronized in time with a common time code.

Digitized video recordings were saved as avi-files and analysed with commercial software (Simi Motion ${ }^{\circledR}$ 6.5,
SIMI Reality Motion Systems GmbH) to reconstruct the bats' 3-D flight path (reconstruction error $\pm 2 \mathrm{~cm}$ ).

The wav-files were cut before their analysis, analysing only the time between the bats' take off from the hand and the time point $20 \mathrm{~ms}$ before landing. With this approach we analysed the approach phase until the end of buzz I and excluded all pulses of buzz II which were sometimes emitted just prior to landing and whose echo information arrives too late for the bats to react to it before contacting the landing grid (Melcón et al. 2007). We did not find differences in the presence of buzz II between treatments, so that our cutoff criterion should not bias the results anyway.

The extraction of pulse parameters from the wav-files was performed using a program written for this purpose 
(Matlab 7, MathWorks, Natick, Massachusetts, United States). The algorithm includes two basic steps: the segmentation of the calls from background noise and the extraction of the desired parameters from the pulses. Pulse segmentation was performed on the spectrograms of the recorded signals. The spectrograms were calculated with an FFT window of 240, Hann window, and an overlap of 0.9, all together providing a resolution of $\Delta t=0.5 \mathrm{~ms}$ and $\Delta f=2 \mathrm{kHz}$. The spectrograms were high-pass filtered in order to exclude low-frequency noise. The segmentation of the pulses from the noise was achieved using a threshold that was applied to the spectrograms with a rectangle moving window of $40 \times 100$ pixels (corresponding to $80 \mathrm{kHz}$ by $50 \mathrm{~ms}$ ). This window-approach enabled us to overcome the temporal-spectral specific noise levels by assuring that the threshold of each rectangle in the spectrogram was defined according to its own noise level. After the pulses were segmented we used several manual techniques in order to make sure that no pulses were left out and no noise was segmented as a pulse.

Once the pulses were extracted from the background noise, the next step of the algorithm extracts their parameters of interest, i.e. pulse duration, starting frequency, best frequency (frequency containing the maximum of energy), and terminal frequency. The beginning and end of each pulse were defined at $25 \mathrm{~dB}$ under their maximum intensity.

To test if the parameters varied along the approach, we computed repeated measures ANCOVA, dividing the distance axis into five classes (1-20, 21-40, 41-60, 61-80, $81-100 \mathrm{~cm})$. To address the questions whether the measured variables changed in the ambiguous scenes, we used one-way ANOVA. For each animal we analysed about 20 trials of each treatment. Statistics were computed with the software JMP 5.1.

\section{Ambiguity detection}

To reduce ambiguity bats could mark consecutive calls by altering any of their echolocation parameters in a way that eases the echo assignment, they can alter the emission pattern to identify virtual targets or in the case of ambiguity type II they can also increase the pulse interval to make sure that all echoes are back before the next signal is emitted. To test the hypothesis of marking the calls we measured the following call parameters: pulse duration, source level (SL), and starting-, best- and terminal frequency. We examined whether the variance observed in the call parameters along the approach was introduced by the bats or was a result of our measuring method (e.g. the directionality of the microphone). If bats marked their signals, we would predict a higher variance in buzz I, where ambiguity is most likely. Therefore, we used a $t$ test to analyse whether the standard deviation of the signal parameters changed from the control behaviour to the test behaviour where the reflector was placed 100 and $250 \mathrm{~cm}$ behind the landing grid.

In RADAR systems virtual echoes are identified by a jitter of the pulse interval from short to long and vice versa thus avoiding constant pulse intervals. This alternation induces a range jitter of the virtual target but not of the real target. We therefore tested if bats use a strategy that increases jitter in response to the more ambiguous scenes. Because the within-group pulse interval is nearly constant and thus groups raise ambiguity, an increase in change of perceived position could be reached by reducing the number of pulses per group. This would lead to a more often alternation between short and constant within-group PIs to long in-between group PIs and vice versa. To measure the effect of ambiguity on the pulse emission pattern, we first counted the number of calls per call group and compared the number of calls in the last call group with an ANOVA and post hoc Dunnett test.

We also measured the percentage of changing PIs created by the sequence, assuming that the more often the object jitters the easier should be to distinguish between the real object and the virtual one. Given that the jittering of the perceived distance of the virtual object is caused by nonidentical consecutive PIs, we calculated the percentage of non-identical consecutive PIs relative to the total number of PI of each sequence. To determine whether the consecutive PIs were identical or non-identical we used a $10 \%$ criterion, meaning that each PI was compared to the previous and following ones and if it was up to $10 \%$ larger or smaller than any of them it was defined as non-identical. Note that the criterion applied here was half of the value used by Moss et al. (2006) to define the sonar strobe groups. We compared the percentage of changing PIs either for the whole sequence or for the last $25 \mathrm{~cm}$ of the approach, where we would expect the bat to undergo ambiguity with higher probability. The statistical test employed to address whether there were significant differences between treatments was $t$ test using a two-side test and correcting (Bonferroni) for multiple testing within each animal. For each animal we analysed 20 trials of each experiment.

To test whether bats increased the PI within the call groups to reduce ambiguity type II we divided the sequences into 8 distance classes of $12.5 \mathrm{~cm}$ each and calculated for each condition the mean of the PI within call groups for each distance class.

\section{System's ensonification}

In order to test whether bats could use spectral cues to determine the range of the object, we ensonified the system with a biomimetic sonar system consisting of a sonar head with three transducers (Polaroid 600 Series; 4-cm-diameter circular aperture) connected to a computer. The sonar head 
was mounted on a portable tripod. Its central transducer served as an emitter (simulating the bat's mouth) and the two side transducers functioned as receivers (simulating the ears). Echoes received from the emitted signal were amplified, A/D converted, and saved by the computer. The emitted signal comprised a 10-ms linear down-sweep from 120 to $25 \mathrm{kHz}$ (similar to a frequency-modulated bat call). We excited the emitter with a constant amplitude, but due to the speakers' frequency response, an unimodal response function was created with a maximum around $50 \mathrm{kHz}$ with a $-20 \mathrm{~dB}$ bandwidth of $\sim 60 \mathrm{kHz}$, providing an intensity of $112 \mathrm{~dB}$ SPL (sound pressure level) at the maximal frequency in a distance of $1 \mathrm{~m}$ from the emitter. The beam width of our emitted pulse was narrower than a typical bats' one, with its first null for $50 \mathrm{kHz}$ occurring around $15^{\circ}$. The recorded echo was digitized at a sampling rate of $1 \mathrm{MHz}$ and with a 12-bit resolution.

To assess potential spectral cues for the discrimination of virtual from real echoes, we simulated two echoic scenes where the bat was replaced by the sonar head which was positioned $30 \mathrm{~cm}$ in front of the landing grid. To test ambiguity type I we compared the frequency response of the reflector when it was $1 \mathrm{~m}$ behind the grid (real object) and $3.3 \mathrm{~m}$ behind the grid (virtual object when PI is $12 \mathrm{~ms}$ ). To test ambiguity type II we compared the frequency response of the reflector when it was $2.5 \mathrm{~m}$ behind the grid (real object) and $0.8 \mathrm{~m}$ behind the grid (virtual object when PI is $12 \mathrm{~ms}$ ). At each position, the reflector was ensonified from 10 different angles, ranging between perpendicular to the reflector and $45^{\circ}$ to both sides (representing typical angles from which the bats would emit their calls according to their flight trajectories). We then compared the averages of those ten spectra between the real and the most probable virtual range.

\section{Results}

\section{Echolocation behaviour}

In either situation-the non-ambiguous with only the landing grid present, or the more ambiguous with an additional reflector either at 1 or $2.5 \mathrm{~m}$ behind the grid-all 4 bats showed generally the typical approach behaviour reported for this species (Melcón et al. 2009). Signals were mainly emitted in groups, with increasing number of calls with decreasing distance to the landing grid (Fig. 2). The terminal group consisted of either buzz I alone or buzz I and buzz II together. In this study we only included the approach behaviour until the end of buzz I in our analysis.

The average pulse duration of all bats decreased along the approach from 1.3 to $0.6 \mathrm{~ms} \quad\left(F_{3,9}=25.3592\right.$, $p=0.0001)$. Average start and best frequency also decreased significantly when bats came closer to the target from 93 to $80 \mathrm{kHz}$ and 65 to $53 \mathrm{kHz}$, respectively $\left(F_{3,9}=\right.$ 13.873 and $F_{3,9}=5.8498, p=0.0010$ and $p=0.0169$, respectively). The terminal frequency, however, did not significantly change along the distance to the landing grid $\left(F_{3,9}=1.3564, p=0.3196\right)$.

\section{Flight behaviour}

The flight paths used by the bats did not change in the ambiguous situations, approaching most times from below in a more or less straight path. The overall flight speed, however, increased significantly in the ambiguous situations in 3 of the 4 bats ( $p<0.0001$ in ambiguous scenes). This could probably be attributed to the fact that these experiments were conducted after the control flights so that the bats were better trained when performing the higher complexity tasks.

\section{Marking calls}

Theoretically bats could prevent ambiguity by acoustically marking the pulses within a group. In search for pulsespecific signatures we examined many different pulse parameters (see experimental procedures) without finding any useful pattern of pulse marking, such as oscillating between high and low values. In addition, none of the parameters showed a significantly enlarged standard deviation in the increased ambiguous situations $(n=20, p>0.2$ in all cases), rejecting the hypothesis that bats mark their pulses in order to ease proper pulse-echo assignment.

\section{PI values}

The analysis of the PIs of control and experimental flights did not reveal remarkable changes. The PI clouds of all 4 bats (Fig. 2a) showed a separation between the longer inbetween group PIs and the shorter within-group PIs. Both were reduced with decreasing distance to the landing site. Close to the landing site bats 1, 3, and 4 encountered the situation with some PIs being shorter than the two-way travel time (TWTT) of echoes from the target at $2.5 \mathrm{~m}$, thus generating a danger of ambiguity type II. Bat 2 which always used shorter pulse intervals encountered this danger throughout the whole approach flight. When comparing the within-group PIs of controls and experimental flights we found almost no difference, especially in the most relevant range from 0.6 to $0 \mathrm{~m}$, when grouping becomes most salient. Only bat 1 reacted with a small increase in PI (Fig. 2b). This indicates that we could not induce a change of the within-group PI by adding the additional targets behind the landing site. 


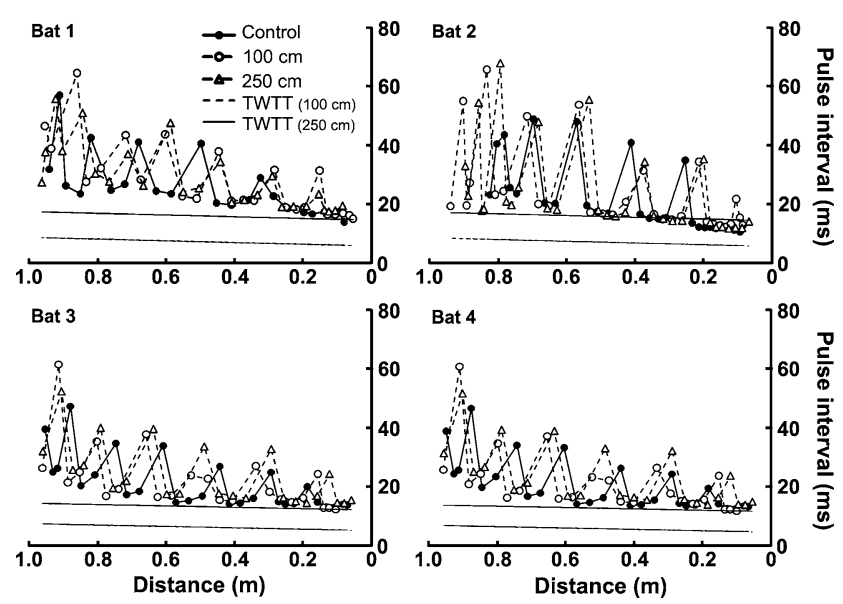

Fig. 3 Representative individual examples of the PI along the distance to the landing grid for the three treatments. The straight lines represent the two-way travel time of the calls for the object at $100 \mathrm{~cm}$ (dashed line) and $250 \mathrm{~cm}$ (solid line). In all bats the last group of the experimental situations was shorter and contained less pulses than in the control

\section{Number of calls}

When comparing pulse patterns of individual bats we found distinct changes towards the end of the approach (Fig. 3). To quantify these changes we compared the average number of pulses per group in the last four groups before landing. Three of the four bats reacted significantly to the presence of the reflectors (100 and $250 \mathrm{~cm}$ ) by reducing the number of calls in the last group (bat 1: $F_{2,61}=11.2148$, $p<0.0001$; bat $2: F_{2,57}=5.4639, p=0.0068$; bat 3 : $F_{2,55}=6.0142, p=0.0043$; bat $4: F_{2,56}=1.7634, p=0.18$ (Fig. 4; Table 1). Post hoc tests revealed a significant decrease $(p<0.05)$ for bats 1,2 , and 3 for both 100 and $250 \mathrm{~cm}$ treatments. In other words, as soon as the reflectors created a more ambiguous echoic scene, the last group of the sequence contained less calls. In the sound groups emitted before the last group the bats did not change the number of pulses per group. Strikingly, in none of the situations the bats changed the overall number of pulses along the approach. Within the last meter before landing they emitted $26 \pm 2$ calls.

\section{Alternating PIs}

To quantify the jitter between long and short PIs within the whole approach sequence, we measured the percentage of calls between non-equal PIs which we will refer to as percentage of alternating PIs. We used the $10 \%$ criterion to define whether the PIs were equal or different. This criterion would not only detect the big PI changes between short within-group PIs and long in-between group PIs but also smaller changes within groups. In three of the four bats the

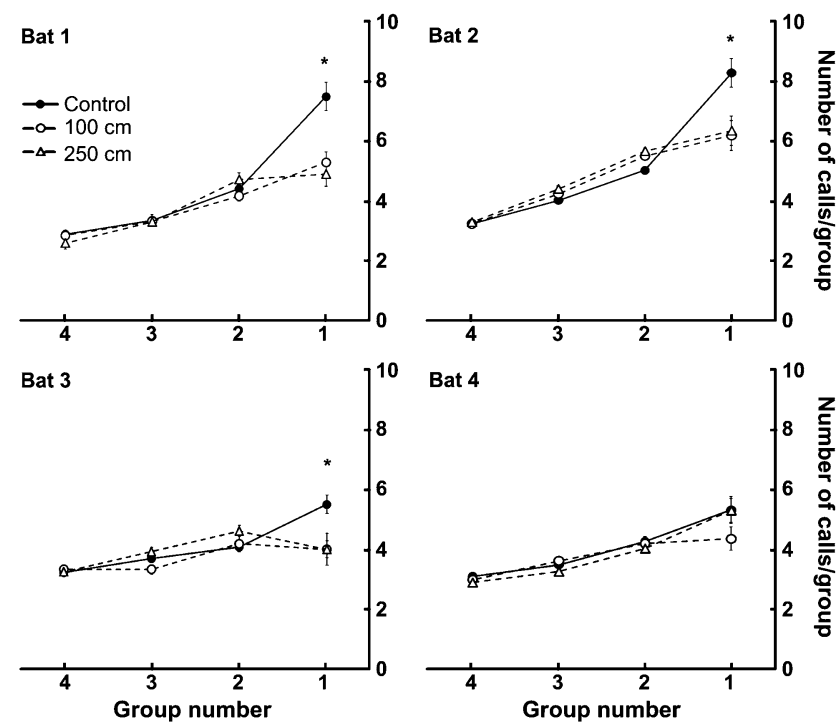

Fig. 4 Average number of calls per group in the last 4 groups before landing and for each bat. Filled circles control, open circles object at $100 \mathrm{~cm}$, open triangles object at $250 \mathrm{~cm}$. Note that in three of the four bats, the number of calls in the last group decreased significantly (asterisk) when the second object was present, either at 100 or $250 \mathrm{~cm}$ behind the grid

Table 1 Mean \pm SEM of the number of pulses for all bats in each call group, where 1 is the last one of the approach, 2 the one before, etc

\begin{tabular}{llll}
\hline $\begin{array}{l}\text { Call group } \\
\text { number }\end{array}$ & Control & $\begin{array}{l}\text { Object } \\
\text { at } 100 \mathrm{~cm}\end{array}$ & $\begin{array}{l}\text { Object } \\
\text { at } 250 \mathrm{~cm}\end{array}$ \\
\hline 1 & $6.7 \pm 0.7$ & $5.0 \pm 0.5$ & $5.1 \pm 0.5$ \\
2 & $4.4 \pm 0.2$ & $4.5 \pm 0.3$ & $4.8 \pm 0.3$ \\
3 & $3.6 \pm 0.2$ & $3.6 \pm 0.2$ & $3.7 \pm 0.3$ \\
4 & $3.1 \pm 0.1$ & $3.1 \pm 0.1$ & $3.0 \pm 0.2$ \\
\hline
\end{tabular}

percentage of alternating PIs value indicated a significant increase in at least one of the ambiguous echoic scenes (Table 2). In general the reaction to the target at $2.5 \mathrm{~m}$ behind the landing site was stronger than to the target in $1.0 \mathrm{~m}$.

\section{Discussion}

Bats adapt their echolocation behaviour to the tasks they perform. A well-studied example is the approach behaviour of landing bats. The changes in echolocation behaviour during the approach have been discussed mainly under the aspect of distance between bat and landing site, and in a few cases also of target strength of the object of interest (Boonman and Jones 2002; Melcón et al. 2007). Here we studied how additional objects behind the landing site influenced the echolocation behaviour of greater mouse-eared bats. Such targets will produce ambiguity, i.e. the bats will 
Table 2 Mean $\pm \operatorname{SD}(n \approx 20)$ percentage of alternating PIs of the whole approach sequences

\begin{tabular}{lllll}
\hline & Bat 1 & Bat 2 & Bat 4 & Bat 4 \\
\hline Control & $60 \pm 7$ & $63 \pm 8$ & $67 \pm 4$ & $58 \pm 5$ \\
Object at $100 \mathrm{~cm}$ & $67 \pm 6(p<0.0001)$ & $67 \pm 6(p=0.25)$ & $67 \pm 6(p=0.90)$ & $61 \pm 8(p=0.20)$ \\
Object at $250 \mathrm{~cm}$ & $67 \pm 9 .(p=0.001)$ & $72 \pm 9(p<0.0001)$ & $70 \pm 8(p=0.09)$ & $62 \pm 5(p=0.05)$ \\
\hline
\end{tabular}

have difficulties to assign returning echoes to the correct emitted signal. Ambiguity type I will occur if the echo of the last pulse is assigned to the former pulse. In this case the range of the resulting virtual target is overestimated. Its position can be calculated by adding the pulse interval to the TWTT of echoes from the real target. Ambiguity type II occurs when the echo of a previous pulse is assigned to the last pulse. This is only possible if the TWTT of echoes from the real target is longer than the PI. Here the range of the resulting virtual target is underestimated. Its virtual position can be calculated by subtracting the pulse interval from the real TWTT. In our scenario with an additional large target at 100 or $250 \mathrm{~cm}$ behind the landing site, the probability for both types of ambiguity increases with decreasing pulse interval (see "Introduction"). In the terminal part of the approach we often found PIs shorter than the TWTT for the target at $2.5 \mathrm{~m}$ which would induce ambiguity type II (Fig. 2a). The probability of ambiguity type I became also more likely towards the end of the approach where pulse intervals are so short that a virtual object beyond the real object may be perceived.

\section{Marking calls}

Theoretically bats have different possibilities to deal with ambiguous situations. A good strategy would be to mark succeeding calls which would ease the proper assignment of echoes (Hiryu et al. 2010). However, we found no change in signal structure in our experimental conditions. Another strategy would be to increase the pulse interval in the presence of further reflectors to make sure that all echoes are back before the next signal is emitted. Again, the bats did not react in this way and did not change the withingroup PI when additional targets were offered (Fig. 2b).

\section{Spectral cues}

Another way to deal with ambiguity is to make use of spectral cues in order to know the range of the real object. To examine whether bats could rely on frequency cues we ensonified the system with the reflector positioned at the real and virtual ranges calculated according to a constant PI of $12 \mathrm{~ms}$. We assumed that if the spectra representing the echoes from the virtual and real objects would be sufficiently different, bats could use them to solve ambiguity.
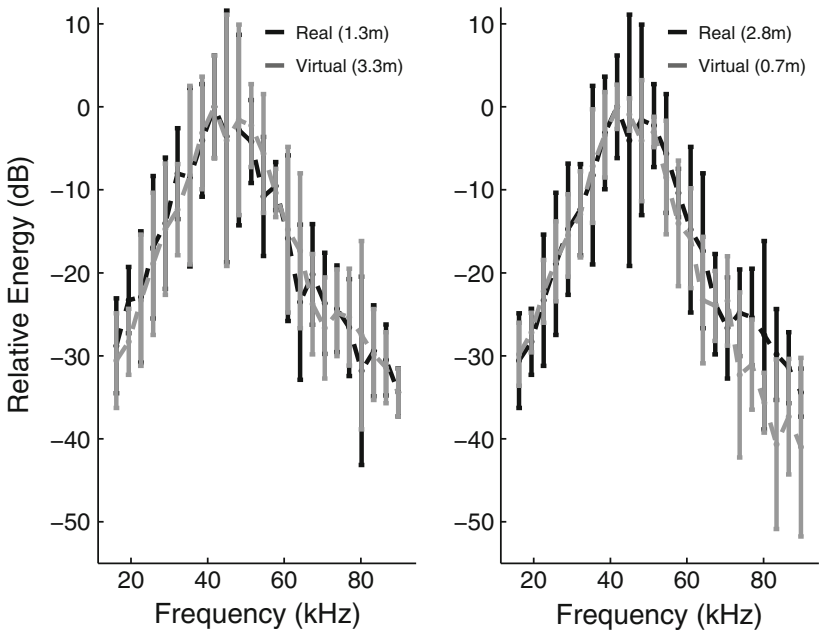

Fig. 5 Mean spectra $\pm \operatorname{SD}(n=10)$ of the echoes returning from the reflector positioned according to the real and virtual ranges calculated when the bat is $30 \mathrm{~cm}$ in front of the grid and calling with a $12 \mathrm{~ms}$ PI. Given the high similarity between the spectra, it is not possible for the bats to rely on spectral cues to discriminate between real and virtual echoes

However, not only did we find a big variability in the echoes spectra with varying impinging angles, but also a high similarity between the mean spectra representing the real and virtual target (Fig. 5).

\section{Alternating PIs}

A further possibility to handle ambiguous situations is a change in pulse temporal pattern as in RADAR systems. The alternation of the pulse interval from short to long and back induces a change in perceived range of the virtual target but not of the real target so that the virtual target can be identified. Our data indicate that bats may use this approach. The presence of additional targets behind the landing site induced distinct changes in the pulse pattern. The number of pulses in the terminal group was significantly reduced from control values often above 8 pulses to 4-6 pulses. The bat which showed no reaction already produced terminal groups with 5-6 pulses in the controls (Fig. 4). Additionally, the experimental conditions induced an increase of the jitter value which indicates more changes in the PI. This effect was more pronounced at the $2.5 \mathrm{~m}$ targets and was significant in 3 animals (Table 2). 
The observed decrease of pulses in the terminal group and the increase in jitter reduces the danger of ambiguity but does not prevent it completely. For instance, in a terminal group with five about equally spaced signals four of them will still indicate virtual targets. However, the bats can somehow deal with this situation. We hypothesize that real and virtual targets are represented on a range map in the brain which is updated after each pulse-echo pair. The reduction of pulses in the terminal group in ambiguous echoic scenes may indicate that a virtual target confirmed by more than $4-5$ succeeding measurements will be evaluated as relevant and will therefore cause problems. Bats can prevent this problem by making groups with lesser pulses which increases the PI jitter. This may also explain why bats only shorten terminal groups with more than six pulses. All earlier groups of an approach sequence contain lesser pulses and are therefore not problematic.

The stronger reaction observed in the alternation of PIs when the object was placed $250 \mathrm{~cm}$ behind the target may indicate that ambiguity type II is more problematic to deal with. An explanation may be that ambiguity type II leads to an underestimation of range, resulting in a virtual target close by. A close object could be perceived by the bat as a potential risk, making it necessary to evaluate whether that object is real. Nevertheless, we also observed a reaction in the number of calls in the last group and in the percentage of alternating PIs (one bat reacted significantly and two showed a trend) when the object was located $100 \mathrm{~cm}$ behind the landing site, where no ambiguity type II was possible. Therefore, we assume that ambiguity type I also plays a role during the approach and that bats deal with it in the same way as with ambiguity type II.

One more argument against ambiguity is that the bats could rely on spatial memory to reach the target. In the current work, given the time bats were trained to perform the task, the constant position of the landing grid, together with the fact that in between trials bats were allowed to fly around and scan the already well-known flight room, the probability that spatial memory played a role was high. However, all bats showed an even stronger response to ambiguity with restricted situations, thus strengthening the idea that ambiguity may indeed be a major issue and therefore the need to double check by, at least under the tested circumstances, altering the control temporal pattern of their echolocation calls.

\section{Ambiguity and background clutter}

Other recent studies (Moss et al. 2006; Petrites et al. 2009) also investigated how bats change their echolocation behaviour when performing a specific task in the presence of background clutter. In the study of Petrites et al. (2009)
E. fuscus had to fly in an open corridor between many clutter targets. The bats reacted to changes in clutter density by emitting groups with more sounds and shorter intervals at higher clutter densities. However, the change of clutter density by adding more clutter targets has little effect on the danger of ambiguity which is only determined by the spatial relation between bat and near targets (ambiguity type I) and the furthest targets (ambiguity type II). Therefore the observed changes cannot be related to changes in ambiguity. Additionally, the bats never used groups with more than four signals which may be not problematic according to our results.

In the study of Moss et al. (2006) Eptesicus fuscus captured tethered insects at different distances to vegetation in the background. This task is difficult to compare with our task. E. fuscus had to solve a detection task first before it approached a small target in front of a close-by large clutter background target whereas our bats approached a large and familiar landing site in front of an additional large target positioned further out. In the experiment with E. fuscus ambiguity type II could not occur as the used PIs were always longer than the TWTT from the clutter target. However, there was a clear chance for ambiguity type I especially at the short PIs in the buzz. This may be an additional explanation for the observed reduction of the buzz II, a component that we did not consider in our own analysis. Interestingly, the number of pulses within the other pulse groups, or strobe groups as they are called by Moss et al. (2006), was also no more than five. Possibly, this number is a trade-off between an increased information flow, necessary during the approach, and the danger of ambiguity.

To further investigate the potential influence of ambiguity on signal pattern, it would be important to know how the bat processes pulse echo trains in groups and why it is a problematic task to deal with more than six signals within a group at ambiguous situations.

Acknowledgments We want to thank Kenneth Wright for revising the English of the work. M. Melcón was funded by the German Academic Exchange Service and Y. Yovel by the human resources and mobility activity Marie Curie host fellowships for early stage research training under contract MEST-CT-2004-504321 PERACT by the European Union. The work was also supported by the German Science Foundation (SFB 550 and Schn 138/27-1). The experiments reported here comply with the current German animal protection law. Bats were caught in Bulgaria (collection permit from the Ministry of Environment and Waters, 34/04.07.2005, Sofia, Bulgaria) and held in the animal facility of the Institute of Neurobiology at the University of Tübingen (approved by the Regierungspräsidium Tübingen, 35-9185.46/Uni Tü).

Open Access This article is distributed under the terms of the Creative Commons Attribution Noncommercial License which permits any noncommercial use, distribution, and reproduction in any medium, provided the original author(s) and source are credited. 


\section{References}

Boonman A, Jones G (2002) Intensity control during target approach in echolocating bats: stereotypical sensori-motor behaviour in Daubenton's bats, Myotis daubentonii. J Exp Biol 205(18):2865-2874

Griffin DR, Webster FA, Michael CR (1960) The echolocation of flying insects by bats. Anim Behav 8:3-4

Hiryu S, Bates ME, Simmons JA, Riquimaroux H (2010) FM echolocating bats shift frequencies to avoid broadcast-echo ambiguity in clutter. PNAS 107(15):7048-7053

Holderied MW, von Helversen O (2003) Echolocation range and wingbeat period match in aerial-hawking bats. Proc R Soc Lond B 270:2293-2299

Holderied MW, Korine C, Fenton BM, Parsons P, Robson S, Jones G (2005) Echolocation call intensity in the aerial hawking bat Eptesicus bottae (Vespertilionidae) studied using stereo videogrammetry. J Exp Biol 208(7):1321-1327

Kalko EKV, Schnitzler HU (1998) How echolocating bats approach and acquire food. In: Kunz TH, Racey PA (eds) Bat biology and conservation. Smithsonian Institution Press, Washington, DC, pp 197-204

Kingston T, Jones G, Zubaid A, Kunz TH (2003) Alternation of echolocation calls in five species of aerial-feeding insectivorous bats from Malaysia. J Mammal 84:205-215

Melcón ML, Denzinger A, Schnitzler H-U (2007) Aerial hawking and landing: approach behaviour in Natterer's bats, Myotis nattereri (Kuhl 1818). J Exp Biol 210:4457-4464
Melcón ML, Schnitzler H-U, Denzinger A (2009) Variability of the approach phase of landing echolocating greater mouse-eared bats. J Comp Physiol A 195(1):69-77

Moss CF, Surlykke A (2001) Auditory scene analysis by echolocation in bats. J Acoust Soc Am 110:2207-2226

Moss CF, Bohn K, Gilkenson H, Surlykke A (2006) Active listening for spatial orientation in a complex auditory scene. PLoS Biol 4(4):615-626

Petrites AE, Eng OS, Mowlds DS, Simmons JA, DeLong CM (2009) Interpulse interval modulation by echolocating big brown bats (Eptesicus fuscus) in different densities of obstacle clutter. J Comp Physiol A 195(6):603-617

Schnitzler H-U, Kalko EKV (2001) Echolocation by insect-eating bats. Bioscience 51(7):557-569

Schnitzler H-U, Kalko E, Miller L, Surlykke A (1987) The echolocation and hunting behavior of the bat, Pipistrellus kuhlii. J Comp Physiol A 161:267-274

Schnitzler H-U, Moss CF, Denzinger A (2003) From spatial orientation to food acquisition in echolocating bats. Trends Ecol Evol 18(8):386-394

Siemers BM, Schnitzler HU (2004) Echolocation signals reflect niche differentiation in five sympatric congeneric bat species. Nature 429:657-661

Skolnik MI (1970) Radar handbook, McGrew-Hill

Wong JG, Waters DA (2001) The synchronisation of signal emission with wingbeat during the approach phase in soprano pipistrelles. J Exp Biol 204:575-583 